\title{
Predictors of cartilage degeneration in patients with subchondral insufficiency fracture of the femoral head: a retrospective study
}

Tomohiro Shimizu ${ }^{\dagger}$, Shunichi Yokota ${ }^{\dagger}$, Yosuke Kimura, Tsuyoshi Asano, Hirokazu Shimizu, Hotaka Ishizu, Norimasa Iwasaki and Daisuke Takahashi ${ }^{*}$

\begin{abstract}
Background: There is evidence that the cause of primary osteoarthritis $(\mathrm{OA})$ is related to the changes in subchondral bone; however, the influence of subchondral insufficiency fracture (SIF) of the femoral head on the degeneration of the hip joint and the prognostic factors related to joint degeneration remain unclear. The objectives of this study were (1) to investigate the natural history of joint space width after the occurrence of SIF and (2) to investigate the associations between joint space narrowing and bone metabolic markers as well as magnetic resonance imaging (MRI) among the patients with SIF.

Methods: Between January 2010 and December 2019, 238 patients in whom band pattern of the femoral head were observed on MRI visited Hokkaido University Hospital. Among these patients, 44 hips in 41 patients were diagnosed with SIF and eligible for this retrospective study. We evaluated the joint space width (JSW) of the hip on the radiograph obtained at the first and last visits, length of the band lesion on MRI, bone mineral density by dualenergy X-ray absorptiometry, and bone metabolism markers. Similarly, the factors associated with the necessity of surgery and the progression of the narrowing of the joint space were evaluated.

Results: Fifteen of the 44 hips required total hip arthroplasty (THA). A significant decrease was observed in the JSW from the first visit to the final follow-up. Changes in the JSW were associated with the length of band patterns, serum type 1 procollagen-N-propeptide (P1NP), and tartrate-resistant acid phosphatase 5b (TRACP-5b) during diagnosis. Additionally, bone metabolic markers tended to be associated with the length of the band pattern.

Conclusions: SIF could cause joint space narrowing and hip OA. In addition to MRI findings as prognostic predictors of SIF, as previously described, bone metabolic markers were equally associated with changes in JSW, suggesting that these parameters could be useful in predicting the prognosis of SIF. Considering that bone metabolic markers trended to be associated with the length of band pattern, they might reflect the local severity.
\end{abstract}

Keywords: Subchondral insufficient fracture, Joint space narrowing, Hip joint, Bone metabolic marker

\footnotetext{
*Correspondence: rainbow-quest@pop02.odn.ne.jp

${ }^{\dagger}$ Tomohiro Shimizu and Shunichi Yokota contributed equally to this work. Department of Orthopaedic Surgery, Faculty of Medicine and Graduate School of Medicine, Hokkaido University, Kita-15 Nishi-7, Kita-ku, Sapporo 060-8638, Japan
}

(c) The Author(s). 2020 Open Access This article is licensed under a Creative Commons Attribution 4.0 International License, which permits use, sharing, adaptation, distribution and reproduction in any medium or format, as long as you give appropriate credit to the original author(s) and the source, provide a link to the Creative Commons licence, and indicate if changes were made. The images or other third party material in this article are included in the article's Creative Commons licence, unless indicated otherwise in a credit line to the material. If material is not included in the article's Creative Commons licence and your intended use is not permitted by statutory regulation or exceeds the permitted use, you will need to obtain permission directly from the copyright holder. To view a copy of this licence, visit http://creativecommons.org/licenses/by/4.0/. The Creative Commons Public Domain Dedication waiver (http://creativecommons.org/publicdomain/zero/1.0/) applies to the data made available in this article, unless otherwise stated in a credit line to the data. 


\section{Background}

Subchondral insufficiency fracture (SIF) of the femoral head has recently been recognized as a cause of the femoral head collapse, resulting in the degeneration of the hip joint, which is known to occur in association with osteonecrosis of the femoral head (ONFH) [1-3]. Although the precise prevalence of SIF is unknown, previous studies with histopathological re-evaluation showed that SIF was observed in 6.3\% (460 of 7349) of patients preoperatively diagnosed with osteoarthritis $(\mathrm{OA})$ and in $11.1 \%$ (41 of 369) of patients with ONFH [4]. Some cases of SIF have been reported to heal after conservative therapy, including rest, non-weight bearing, and traction $[1$, $5,6]$, whereas other cases have been reported to undergo collapse necessitating surgery such as total hip arthroplasty (THA) and osteotomy $[2,3,7,8]$. On the contrary, although there is evidence that the cause of primary OA is related to the changes in subchondral bone $[9,10]$, the influence of SIF on the degeneration of the hip joint and the prognostic factors related to joint degeneration among patients with SIF remain unclear.

SIF has been reported in adults of varying ages and activity levels [11-13]. Previous studies reported that the risk factors for THA were female sex [14], elderly onset [15], and length [16], location [17], and luminance of band pattern [18]. On the contrary, although bone fragility due to osteoporosis could be considered the most important cause of SIF, similar to vertebral body fractures [19], another study reported no differences in bone mass densitometry (BMD) between patients with and without (controls) SIF [20]. Bone strength is determined by bone mass, geometry, and quality, including bone turnover, microarchitecture, and the degree and distribution of mineralization [21]. Among these, one review article reported a variation in the levels of bone turnover markers throughout the course of fracture repair, which was dependent on the size of the fracture and the healing time [22]. Therefore, evaluating whether and how bone turnover markers would affect the pathology of SIF would be of significant interest.

Hence, the objectives of this study were (1) to investigate the natural history of joint space width after the occurrence of SIF and (2) to investigate the associations between joint space narrowing and bone metabolic markers as well as magnetic resonance imaging (MRI). The hypotheses of this study were as follows: (1) SIF could induce joint space narrowing and hip OA, and (2) bone metabolic abnormalities and MRI findings could predict the prognosis and reflect the severity of SIF.

\section{Patients and methods}

The Institutional Review Board approved this retrospective study (\# 015-0206). In total, 238 patients (401 hips) who showed a band pattern of the femoral heads on
MRI visited Hokkaido University Hospital from January 2010 to December 2019. Among these, SIF was diagnosed based on several published criteria [2, 23, 24]: hip pain that manifested without any apparent history of trauma; radiographs that were normal or indicated a collapse of the femoral head, joint space narrowing, and/or a linear patchy sclerotic area in the superior portion of the femoral head; a bone marrow edema pattern in the femoral head and/or neck on MRI; and a subchondral low signal-intensity band on T1-weighted MRI that was convex to the articular surface and parallel to the subchondral bone end-plate. We distinguished between SIF and ONFH via gadolinium-enhanced MRI. Forty-seven hips in 44 patients (male 10, female 34) were diagnosed with SIF. In this study, 3 hips in 3 patients who demonstrated rapid collapse and joint destruction, including that of the acetabular (AC), were excluded (Fig. 1). Power analysis was performed to detect a $10 \%$ difference in the survival rate between patients with and without MRI positive findings (intensity change area) with a power of $80 \%$ at a significance level of 0.05 . Thirty-three subjects were needed, and we could recruit a sufficient number of patients for this study.

Among those diagnosed with SIF, patients who could be diagnosed within 3 months after hip pain were supervised to avoid weight-bearing with crutches for 6 weeks [25] and were treated on an outpatient basis every 2 weeks. Patients with late diagnosis or poor compliance could not be initially treated with conservative therapy.

Data on patient demographics, including age, sex, and body mass index (BMI); the period from onset to the first visit; history of corticosteroid intake or alcohol abuse; and medical history of osteoporosis drug intake were collected from their medical records. Data regarding the possible treatment of patients with conservative therapy and the requirement of THA within the follow-up period were similarly collected. The indication of THA was persistent pain and disability of daily life activities regardless of nonsteroidal anti-inflammatory medications. Alcohol abuse was defined as the consumption of more than $400 \mathrm{~mL}$ of alcohol per week, which is known to be a significant risk factor for osteonecrosis of the femoral head [26].

Radiographs were taken using a similar technique throughout the study period; a standardized position of the beam and radiographic penetration were adopted. The radiographs of all patients were assessed using a picture archiving and communication system (PACS) on the anteroposterior (AP) radiographs. In this study, the center-edge (CE) angle at the first visit and longitudinal joint space width (JSW) were investigated (Fig. 2a). In JSW analysis, concentric circles passing through three points set arbitrarily in the $\mathrm{AC}$ joint surface and the femoral head were drawn (circle $\mathrm{A}$ and circle $\mathrm{B}$ in Fig. 2a). The distance between the intersection of each 

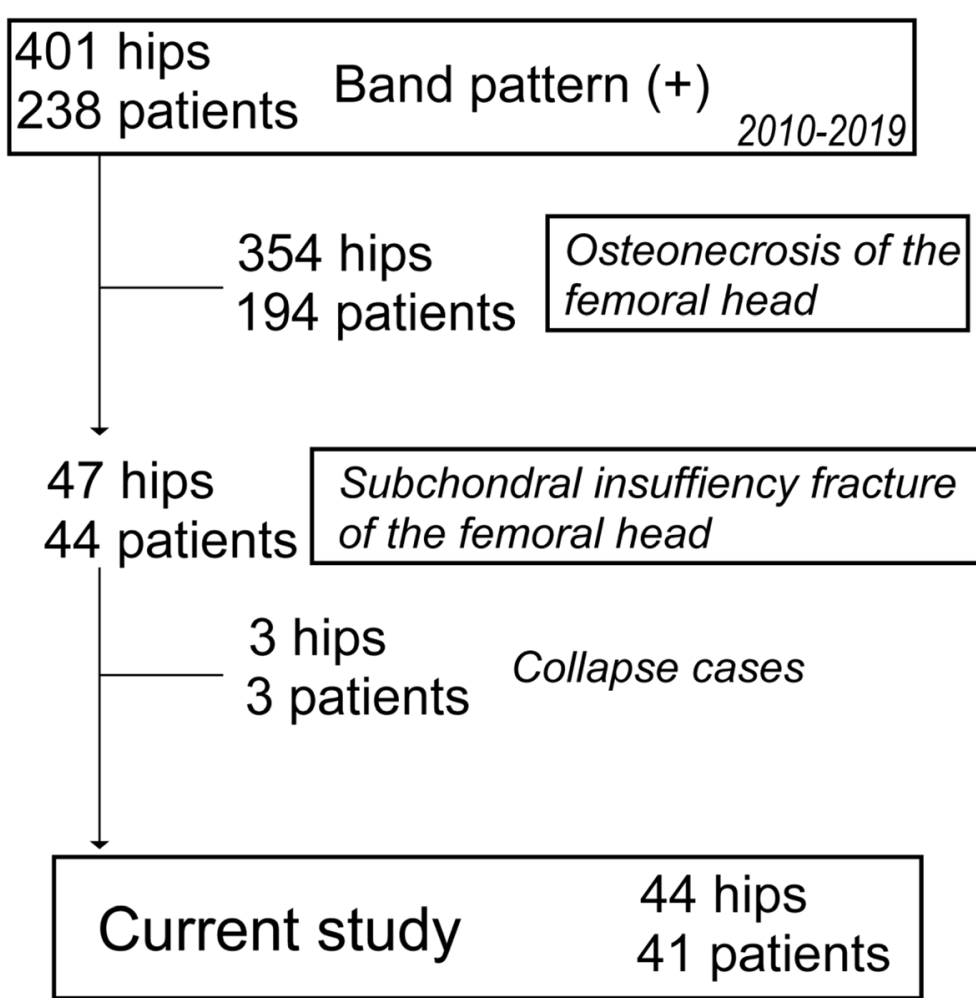

Fig. 1 Flow diagram of study subjects

circle and the line that runs through the center of the femoral head (O in Fig. 2a) perpendicular to the line between the bilateral teardrops (line A in Fig. 2a) was measured. The interobserver variability in the JSW between two observers (YK and TD) was 0.768 .
In this study, the MRI examinations were performed using a 1.5 - $\mathrm{T}$ system under $5-\mathrm{mm}$ slice thickness within 1 week after the first visit to hospital. The T1- and T2weighted spin-echo images and short tau inversion recovery (STIR) images on the coronal and axial (and/or
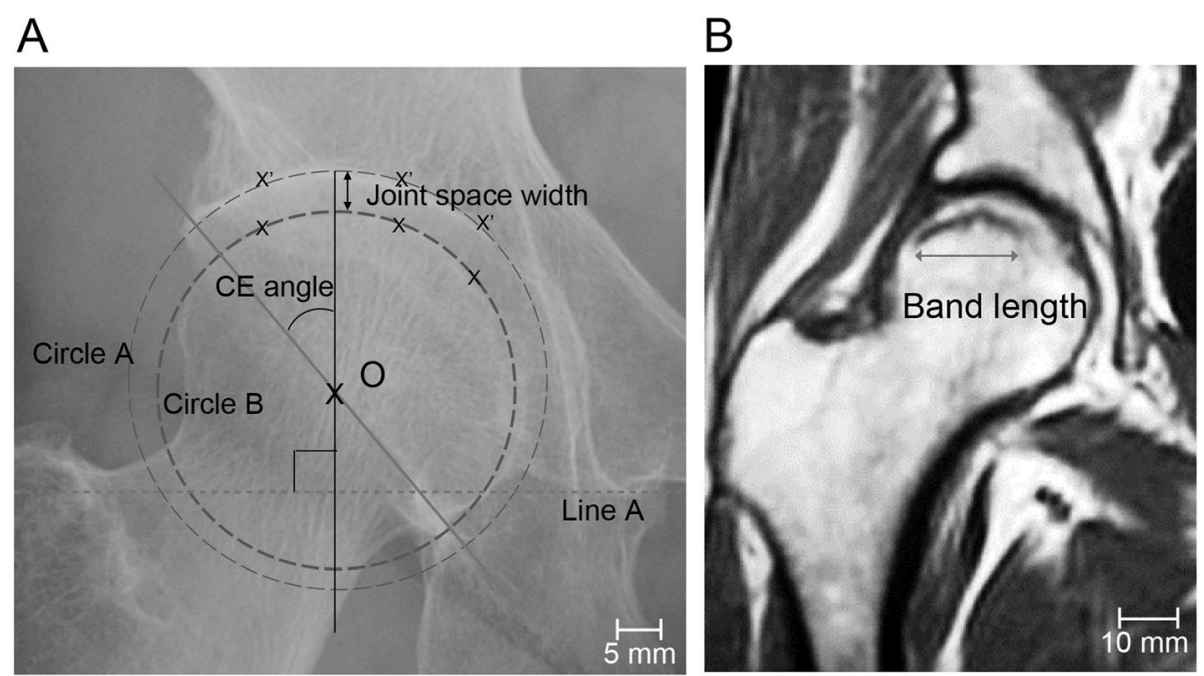

Fig. 2 Radiological evaluation. a The radiographic indices used for the evaluation of the hip are shown. $O=$ center of the femoral head; Line $A=$ line between the teardrops on both sides; Circle $\mathrm{A}=$ circle passing through three points set arbitrarily in the acetabular joint surface; Circle $\mathrm{B}=$ circle passing through three points set arbitrarily in the femoral head. Scale bar, $5 \mathrm{~mm}$. b T1-weighted magnetic resonance imaging (MRI) image illustrating the method used to measure the length of the low-intensity band. Scale bar, $10 \mathrm{~mm}$. CE, center-edge 
oblique axial: paralleling the femoral neck axis) planes were available in all cases. The band lengths were measured at the slice wherein the longest band was detected on the T1-weighted MRI (on the coronal plane), as previously described [16] (Fig. 2b). The interobserver variability in the band length between two observers (YK and TD) was 0.836 .

Fasting blood samples were obtained to examine the biochemical markers of osteoporosis-related bone turnover, including the levels of intact type 1 procollagen-Npropeptide (P1NP) and tartrate-resistant acid phosphatase 5b (TRACP 5b). Because type I collagen-derived peptides, such as CTX-1 (cross-linked C terminal telopeptides of type I collagen) and NTX-1 (cross-linked Ntelopeptide of type I collagen) are excreted through the kidneys, they can be affected by renal dysfunction; therefore, this study evaluated the serum levels of P1NP and TRACP-5b. Serum P1NP level was measured using electrochemiluminescence immunoassay, whereas serum TRACP-5b level was measured using enzyme immunoassay (SRL, Inc. Tokyo). Areal BMD in the lumbar spine (LS, L2-L4) and femoral neck was assessed by dualenergy X-ray absorptiometry (DXA; Discovery A, Hologic Japan, Inc., Tokyo, Japan). Bone turnover markers and $\mathrm{BMD}$ were investigated during the definite diagnosis following the acquisition of MRI images.

Chi-squared or independent $t$ tests were used to compare the differences between patients who required THA and those treated conservatively and between patients who could comply with the weight-bearing limitation and those who could not. Cox regression analysis was performed to identify the risk factors for THA. Linear regression models were adjusted for age, sex, BMI, and anti-osteoporosis therapy; in addition, the period from onset to diagnosis was established to determine the associations between changes in the JSW, band length, and bone metabolic markers. All statistical analyses were performed using SPSS Statistics version 23.0 (IBM Corporation, Armonk, NY); values of $p$ less than 0.05 were considered statistically significant.

\section{Results}

The demographics and clinical data of the patients are summarized in Table 1. In total, 18 of 44 hips could complete the weight-bearing limitation for 6 weeks. Six hips that could not complete the weight-bearing limitation were the late diagnosis cases. Of 7 patients who had undergone anti-osteoporosis therapy, one patient was treated monthly with minodronic acid and the others were treated by active vitamin D3. Fifteen (14 patients) of 44 hips required THA. The mean period from the diagnosis of SIF to THA was 10.5 months (range; 2-54 months). Ten hips (9 patients) developed hip dysplasia $(\mathrm{CE}<20$ degrees).
Table 1 Patient demographics

\begin{tabular}{ll}
\hline 41 patients, 44 hips & \\
\hline Age, years & $61.6(2.3)$ \\
Sex, male to female & $8: 33$ \\
Body mass index, $\mathrm{kg} / \mathrm{m}^{2}$ & $25.5(0.8)$ \\
Period from onset to first visit, months & $2.2(0.4)$ \\
Follow-up period, months & $26.6(2.3)$ \\
Glucocorticoid use, cases & 9 \\
Alcohol abuse, cases & 6 \\
Anti-osteoporosis therapy, cases & 7 \\
Weight-bearing limitation, hips & 18 \\
Total hip arthroplasty, hips & 15 \\
\hline
\end{tabular}

Data are represented as the mean (standard error of the mean)

The JSW in the ipsilateral side demonstrated a significant decrease from the first visit to the final follow-up $(P<0.001)$ (Fig. 3). No significant difference was observed in the JSW from the first visit to the final followup on the contralateral side. The changes in the JSW on the ipsilateral side were associated with the length of the band pattern $(\beta=0.499, P<0.001)$ (Fig. 4a), serum P1NP (Fig. 4b) $(\beta=0.564, P<0.001)$, and TRACP 5b (Fig. 4c) $(\beta=0.452, P=0.004)$. Additionally, the serum P1NP and TRACP $5 \mathrm{~b}$ levels tended to be associated with the length of the band pattern $(\beta=0.376, P=0.035$, and $\beta=0.268$, $P=0.140$, respectively) (Fig. $5 \mathrm{a}$, b).

The comparisons of clinical and radiological findings between patients who could complete the weightbearing limitation therapy (WB limitation) and could not (non-WB limitation) are summarized in Table 2 . WB limitation group showed a younger age, shorter

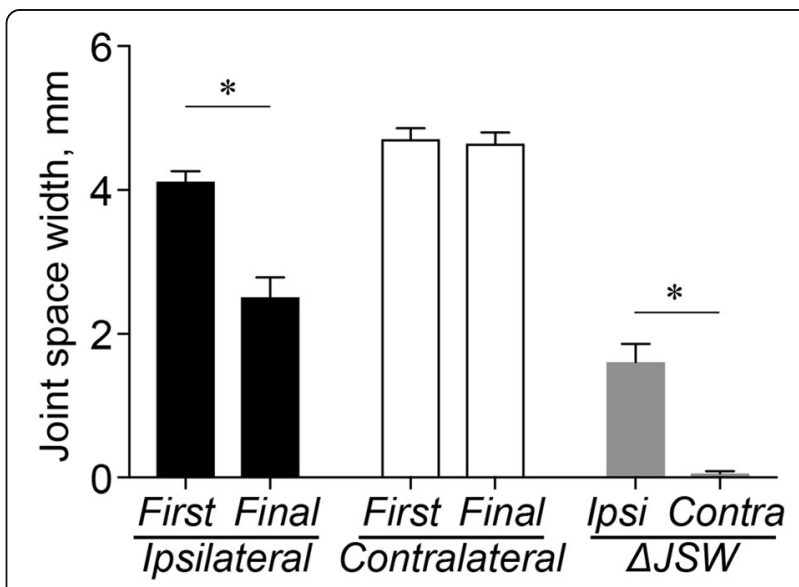

Fig. 3 Comparisons of joint space width. Mean joint space width and change in joint space width from the first visit to the final follow-up in the ipsilateral and contralateral sides. Ipsi, ipsilateral; Contra, contralateral; JSW, joint space width. Asterisks indicate $P<0.05$ 

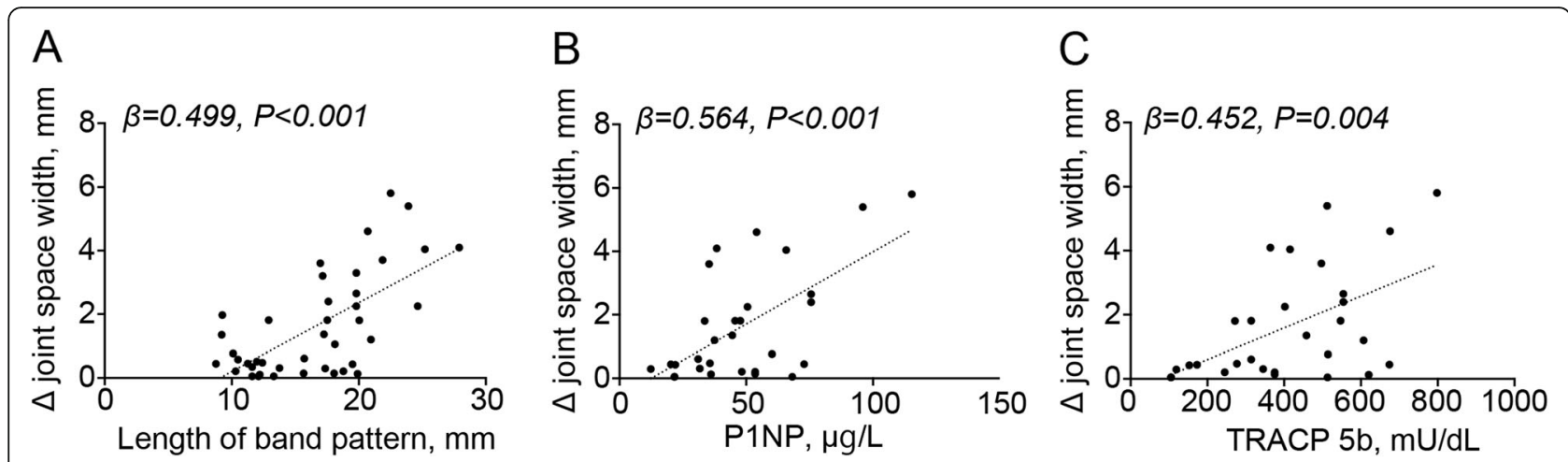

Fig. 4 Association with the changes in joint space width. Scatter plot of changes in joint space width versus a length of band pattern, $\mathbf{b}$ type 1 procollagen-N-propeptide, and c tartrate-resistant acid phosphatase 5b. P1NP, type 1 procollagen-N-propeptide; TRACP 5b, tartrate-resistant acid phosphatase $5 b$

period from pain onset to visit, lower ratio of THA, and smaller changes in JSW than non-WB limitation group.

The comparisons of the clinical findings, image evaluation, and bone metabolic markers between patients who underwent THA (THA group) and who did not undergo THA (non-THA group) are summarized in Table 3 . The non-THA group was younger $(P=0.028)$ and had a shorter period from pain onset to visit $(P=0.002)$ and higher ratio of weight-bearing limitation $(P=0.042)$ than the THA group. No significant differences in JSW and CE angle at the first visit were observed between the two groups. The THA group exhibited a more significant change in JSW from the first visit to the final follow-up than the non-THA group $(P<0.001)$ (Fig. 6a, c, d, f). In addition, the THA group exhibited longer band length $(P<0.001)$ (Fig.6b, e), a higher ratio of the existence of band over the edge of $\mathrm{AC}$ $(P=0.006)$, and a more significant intensity change in AC $(P<0.001)$ than the non-THA group. Although no significant differences were observed in BMD between both groups, the THA group exhibited higher P1NP $(P=0.002)$ and TRACP $5 \mathrm{~b}(P=0.001)$ levels than the non-THA group.
In the univariate analyses, age, period from pain onset to visit, MRI findings, and bone metabolic markers were identified as predictors of THA. Furthermore, MRI findings were identified as the potential predictors of THA in a Cox proportional hazard model adjusted for age, sex, BMI, anti-osteoporosis therapy, and the period from pain onset to visit (Table 4) (Fig. 7).

\section{Discussion}

This study exhibited that JSW significantly decreased from the first visit to the final follow-up and approximately one third of patients progressed to THA; this suggest that SIF could cause hip OA. Since some cases of SIF resolved by weight-bearing limitation [2, 27, 28], nonoperative treatment would be the first choice in all patients. Although the multivariate Cox-regression analysis in this study showed that weight-bearing limitation could not prevent THA, it could reduce the progression of joint space narrowing. Additionally, considering that age and the period from pain onset to visit were significantly different between the WB and non-WB limitation
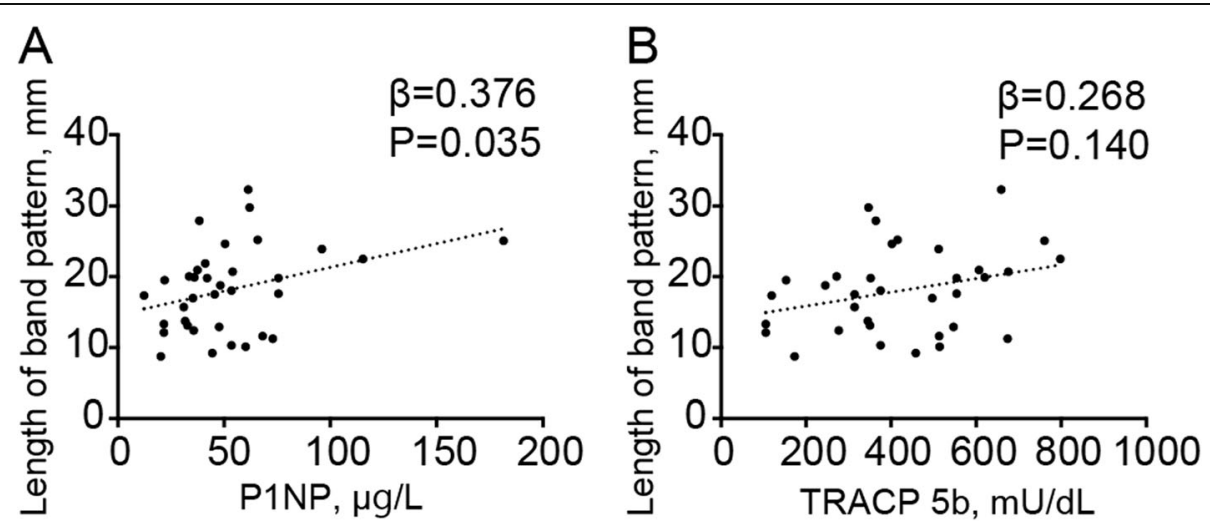

Fig. 5 Association between the length of band pattern and bone metabolic markers. Scatter plot of the length of band pattern versus a type 1 procollagen-N-propeptide and $\mathbf{b}$ tartrate-resistant acid phosphatase $5 \mathrm{~b}$. P1NP, type 1 procollagen-N-propeptide; TRACP 5b, tartrate-resistant acid phosphatase $5 b$ 
Table 2 Comparisons between patients who could complete weight-bearing limitation and those who could not

\begin{tabular}{llll}
\hline & WB limitation (18 hips) & Non-WB limitation (26 hips) & $\boldsymbol{P}$ value \\
\hline Male to female & $5: 13$ & $3: 23$ & 0.170 \\
Age, years & $53.8(4.3)$ & $67.2(2.0)$ & $\mathbf{0 . 0 0 3}$ \\
$\mathrm{BMI}, \mathrm{kg} / \mathrm{m}^{2}$ & $26.4(1.8)$ & $25.1(1.1)$ & 0.525 \\
Period from pain onset to visit, months & $1.1(0.2)$ & $2.5(0.4)$ & $\mathbf{6}(22.2 \%)$ \\
Osteoporosis therapy, case (\%) & $1(5.6 \%)$ & $12(46.2 \%)$ & $\mathbf{0 . 0 0 8}$ \\
Total hip arthroplasty, case (\%) & $3(16.7 \%)$ & & $\mathbf{0 . 1 1 8}$ \\
Radiography findings & & $4.25(0.21)$ & $\mathbf{0 . 0 4 2}$ \\
$\quad$ JSW at the first visit, mm & $4.15(0.19)$ & $2.24(0.36)$ & $\mathbf{0 . 7 8 5}$ \\
Changes in JSW, mm & $1.12(0.36)$ & $24.2(1.4)$ & $\mathbf{0 . 0 3 9}$ \\
Center-edge angle, degree & $25.9(1.6)$ & 0.428
\end{tabular}

Data are represented as the mean (standard error of the mean)

$W B$ weight-bearing, $B M I$ body mass index, JSW joint space width, $A C$ acetabulum

groups, the association with THA, early detection, and treatment are important for SIF. There might be some cases, such as older patients with impaired vision or low balance activity, who require treatment on an inpatient basis, rather than an outpatient basis.

The study findings showed that bone metabolic markers and the length of band patterns were associated with the changes in JSW, suggesting that these parameters could be predictors of poor progression in patients with SIF. The MRI findings of this study were consistent with those of previous reports regarding the association of clinical outcome and MRI [16, 25], suggesting that MRI findings, including the length of band pattern and location of intensity change during diagnosis, could be prognostic predictors as well as highly sensitive indicators for SIF investigation. In addition, serum

Table 3 Comparisons between patients who received total hip arthroplasty and did not

\begin{tabular}{|c|c|c|c|}
\hline & THA (15 hips) & Non-THA (29 hips) & $\boldsymbol{P}$ value \\
\hline Male to female & $3: 12$ & $5: 24$ & 0.822 \\
\hline Age, years & $69.6(2.2)$ & $57.8(3.1)$ & 0.014 \\
\hline $\mathrm{BMI}, \mathrm{kg} / \mathrm{m}^{2}$ & $24.9(1.5)$ & $26.0(1.4)$ & 0.593 \\
\hline Osteoporosis therapy, case (\%) & $2(13.3 \%)$ & $5(17.2 \%)$ & 0.737 \\
\hline Period from pain onset to diagnosis, months & $3.8(1.0)$ & $1.3(0.2)$ & 0.002 \\
\hline Weight-bearing limitation, case (\%) & $3(20.0 \%)$ & $15(51.7 \%)$ & 0.042 \\
\hline \multicolumn{4}{|l|}{ Radiography findings } \\
\hline JSW at the first visit, $\mathrm{mm}$ & $4.26(0.28)$ & $4.18(0.18)$ & 0.793 \\
\hline Changes in JSW, mm & $3.79(0.36)$ & $0.75(0.15)$ & $<0.001$ \\
\hline Center-edge angle, degree & $25.3(1.6)$ & $24.7(1.4)$ & 0.786 \\
\hline \multicolumn{4}{|l|}{ MRI findings } \\
\hline Band length, mm & $23.3(1.1)$ & $14.2(0.7)$ & $<0.001$ \\
\hline Band over the edge of AC, case (\%) & $10(66.7 \%)$ & $7(24.1 \%)$ & 0.006 \\
\hline Intensity change of AC, case (\%) & $14(93.3 \%)$ & $6(20.7 \%)$ & $<0.001$ \\
\hline \multicolumn{4}{|l|}{ YAM, \% } \\
\hline Lumbar & $93.9(4.8)$ & $92.6(3.6)$ & 0.824 \\
\hline Femoral neck & $80.2(2.7)$ & $82.0(2.3)$ & 0.632 \\
\hline \multicolumn{4}{|l|}{ Bone metabolic marker } \\
\hline $\mathrm{P} 1 \mathrm{NP}, \mu \mathrm{g} / \mathrm{ml}$ & $73.0(11.1)$ & $39.7(3.7)$ & 0.002 \\
\hline TRACP 5b, mU/dL & $549.7(40.6)$ & $342.4(38.1)$ & 0.001 \\
\hline
\end{tabular}

Data are represented as the mean (standard error of the mean)

THA total hip arthroplasty, BMI body mass index, JSW joint space width, AC acetabulum, YAM young adult mean, $P 1 M P$ intact type 1 procollagen-N-propeptide, TRACP $5 b$ tartrate-resistant acid phosphatase $5 \mathrm{~b}$ 

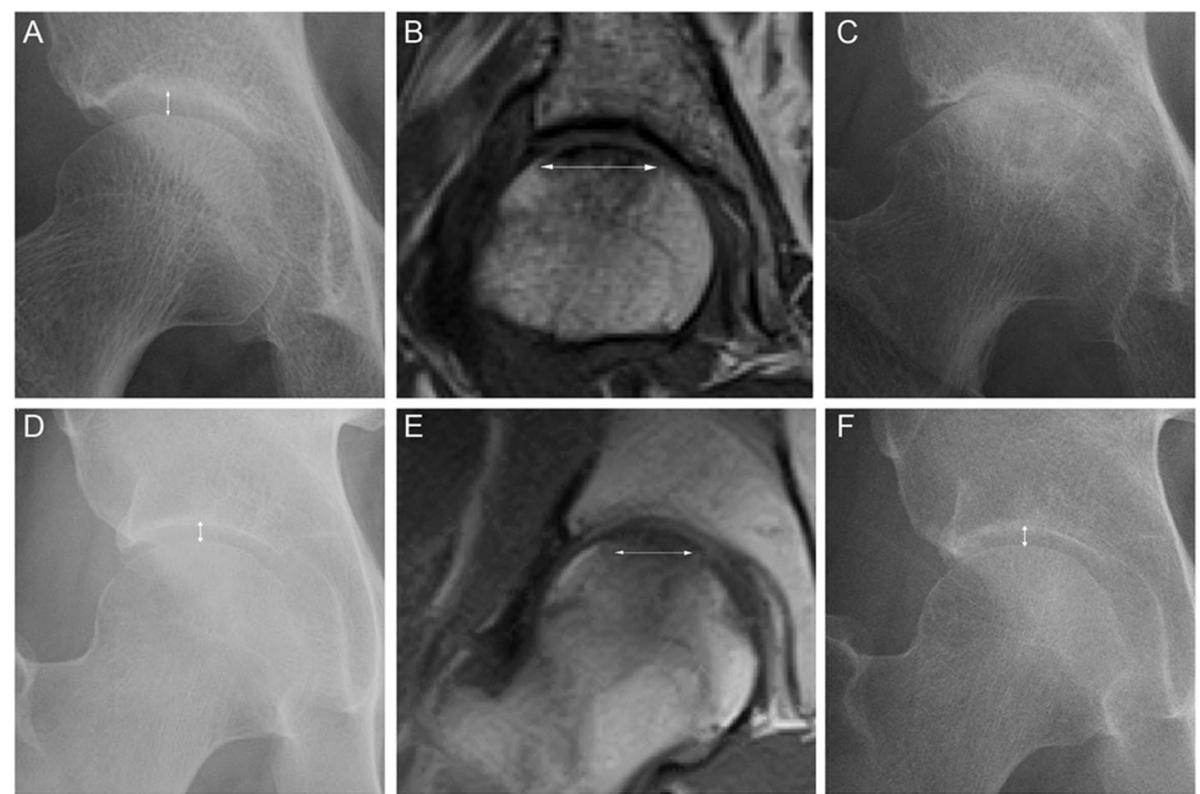

Fig. 6 Longitudinal radiological images in patients who underwent total hip arthroplasty and those who did not. a Anteroposterior (AP) radiograph of the hip at the first visit in a 68-year-old female with hip pain. White arrow represents joint space width. $\mathbf{b}$ Coronal T1-weighted magnetic resonance image (MRI) of the hip at the same week. White arrow represents length of low-intensity band pattern in the femoral head. c AP radiograph of the hip at the final follow up. Joint space narrowing progressed, and she underwent total hip arthroplasty. $\mathbf{d}$ AP radiograph of the hip at the first visit in a 47-year-old male with hip pain. White arrow represents joint space width. e Coronal T1-weighted MRI of the hip at the same week. White arrow represents length of low-intensity band pattern in the femoral head. $\mathbf{f}$ AP radiograph of the hip at the final followup (3 years after the first visit). Patient demonstrated no progression in joint space narrowing

Table 4 Univariate and multivariate Cox-regression analysis for the predictors of total hip arthroplasty in patients with subchondral insufficient fracture of the femoral head

\begin{tabular}{|c|c|c|c|c|c|c|}
\hline \multirow[t]{2}{*}{ Variables } & \multicolumn{3}{|c|}{ Univariate analysis } & \multicolumn{3}{|c|}{ Multivariate analysis } \\
\hline & $P$ value & $H R$ & $95 \% \mathrm{Cl}$ & $P$ value & $H R$ & $95 \% \mathrm{Cl}$ \\
\hline Sex & 0.493 & 1.56 & $0.438-5.557$ & & & \\
\hline Age & 0.020 & 1.07 & $1.011-1.129$ & & & \\
\hline BMl & 0.659 & 0.98 & $0.889-1.077$ & & & \\
\hline Osteoporosis therapy & 0.665 & 0.72 & $0.161-3.208$ & & & \\
\hline Period from onset to diagnosis & 0.021 & 1.28 & $1.038-1.578$ & & & \\
\hline Weight-bearing limitation & 0.107 & 0.35 & $0.099-1.251$ & 0.727 & 0.77 & $0.179-3.324$ \\
\hline \multicolumn{7}{|l|}{ MRI findings } \\
\hline Band length & $<0.001$ & 1.30 & $1.152-1.455$ & 0.001 & 1.29 & $1.116-1.495$ \\
\hline Band over the edge of AC & 0.014 & 3.87 & $1.317-11.375$ & 0.042 & 3.66 & $1.049-12.803$ \\
\hline Intensity change of $\mathrm{AC}$ & 0.001 & 28.85 & $3.677-226.286$ & 0.007 & 29.15 & $2.517-337.594$ \\
\hline \multicolumn{7}{|l|}{ Bone metabolic marker } \\
\hline $\mathrm{P} 1 \mathrm{NP}, \mu \mathrm{g} / \mathrm{ml}$ & 0.018 & 1.013 & $1.002-1.024$ & 0.148 & 1.01 & $0.997-1.023$ \\
\hline TRACP 5b, mU/dL & 0.007 & 1.005 & $1.001-1.008$ & 0.114 & 1.00 & $0.999-1.008$ \\
\hline
\end{tabular}

Multivariate analyses were adjusted for sex, age, BMI, anti-osteoporosis therapy, and period from pain onset

$H R$ hazard ratio, $C l$ confidence interval, $B M I$ body mass index, $A C$ acetabulum, $P 1 N P$ intact type 1 procollagen-N-propeptide, TRACP $5 b$ : tartrate-resistant acid phosphatase $5 \mathrm{~b}$ 

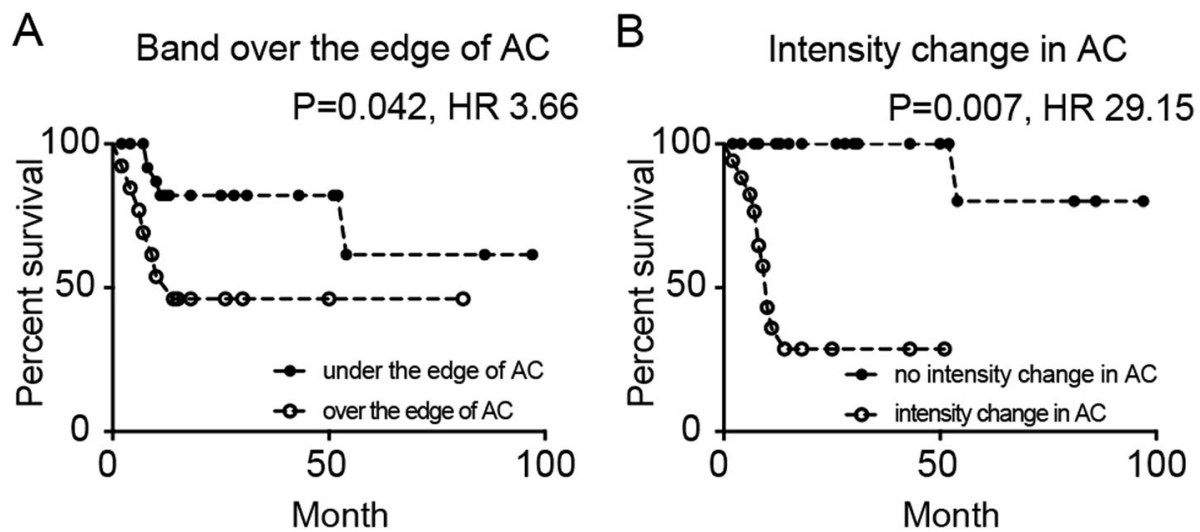

Fig. 7 Kaplan-Meier curves for the requirement of total hip arthroplasty. Kaplan-Meier curves for the requirement of total hip arthroplasty (THA) between a patients with and without intensity changes in the acetabulum and $\mathbf{b}$ patients in whom the band pattern ended and was under the edge of the acetabulum. AC, acetabulum; HR, hazard ratio

P1NP and TRACP-5b were associated with joint space narrowing, suggesting that the levels of bone metabolic markers might be predictors for OA among patients with SIF. Considering that serum P1NP and TRACP-5b tended to be associated with the length of band pattern, bone metabolic markers might reflect the severity of fracture. On the contrary, because this study investigated only serum P1NP and TRACP-5b, future study should address the mechanism underlying the association of other bone turnover markers, such as bone specific alkaline phosphatase, osteocalcin, CTX-1, and NTX-1 with joint space narrowing in patients with SIF.

While numerous groups reported the occurrence of SIF in patients with osteoporosis $[1,2,6,7]$, this study showed that mean BMD did not fulfill the diagnostic criteria for osteoporosis. On the contrary, in some reports on young adults and adolescents, the activity levels did not correspond to a high occurrence of SIF [12, 29]. Therefore, the mechanisms of occurrence and joint space narrowing associated with osteoporosis in patients with SIF remain unclear. Contrary to findings in previous reports, the mean $\mathrm{CE}$ angle observed in this study did not fulfill the criteria for a dysplastic hip $\left(\mathrm{CE}<20^{\circ}\right)$ [20]. In addition, the CE angle in this study was not associated with the changes in JSW and clinical outcomes. A recent study reported that SIF with pre-collapse was associated with bony deformities and lateral labral tears [30]. Therefore, the instability of the hip joint induced by abnormal morphologies, such as dysplasia and femoroacetabular impingement, might affect the occurrence and prognosis of SIF. Although this study did not obtain radial MRI or investigate AC labral tears, future studies should address the association between labral tears and the prognosis of SIF.

There are some limitations to this study. First is the timing of the occurrence of SIF. Since SIF usually occurs without a history of trauma, it is challenging to clarify the accurate timing of the occurrence. The second is the method of JSW measurement. This study investigated the JSW following the technique shown in Fig.1a, and we believed that this method was highly reproducible. However, future studies should address the JSW measurement via computer-based radiographic quantification. The third is the short follow-up duration (mean of 25.4 months) of JSW. Therefore, future studies are warranted to verify the relation of prognostic predictors to the changes in JSW in longer follow-up periods.

\section{Conclusion}

In conclusion, SIF could cause joint space narrowing and hip OA. In addition to MRI findings as prognostic predictors of SIF, as previously described, bone metabolic markers were equally associated with the changes in JSW, suggesting that these parameters could be useful in predicting the prognosis of SIF. Considering that bone metabolic markers tended to be associated with the length of band pattern, they might reflect the local severity.

\section{Abbreviations \\ AC: Acetabular; AP: Anteroposterior; BMD: Bone mineral density; BMI: Body mass index; CE: Center-edge; CTX: Cross-linked C terminal telopeptides of type I collagen; JSW: Joint space width; MRI: Magnetic resonance imaging; NTX: Cross-linked N-telopeptide of type I collagen; OA: Osteoarthritis; ONFH: Osteonecrosis of the femoral head; PACS: Picture archiving and communication system; P1NP: Type 1 procollagen-N-propeptide; SIF: Subchondral insufficiency fracture of the femoral head; THA: Total hip arthroplasty; RACP-5b: Tartrate-resistant acid phosphatase 5b}

\section{Acknowledgements}

We would like to thank Editage (www.editage.com) for English language editing.

\section{Authors' contributions}

TS and YS equally contributed to the data collection, performed the analysis, interpreted the results, and wrote the manuscript. YK, TA, HS, and HI 
contributed to the data collection, interpretation of the result, and technical coordination. $\mathrm{NI}$ contributed to the design of the study and reviewed the manuscript. TD contributed to the design of the study and interpretation of the result and reviewed the manuscript. Approval of the final version of the manuscript was obtained from all authors.

\section{Funding}

The study was supported by a Grant-in-Aid for Young Scientists from the Ministry of Education, Culture, Sports, Science, and Technology of Japan 17 K16670 and 20K17984.

\section{Availability of data and materials}

The datasets used and/or analyzed during the present study are available from the corresponding author on reasonable request.

\section{Ethics approval and consent to participate}

The Institutional Review Board for Human Research approved this study design (\# 015-0206)

\section{Consent for publication}

Not applicable.

\section{Competing interests}

The authors declare that they have no competing interests.

Received: 20 April 2020 Accepted: 9 June 2020

Published online: 22 June 2020

\section{References}

1. Bangil M, Soubrier M, Dubost JJ, Rami S, Carcanagues Y, Ristori JM, Bussiere $J$ L. Subchondral insufficiency fracture of the femoral head. Rev Rhum Engl Ed. 1996:63(11):859-61.

2. Yamamoto T, Bullough PG. Subchondral insufficiency fracture of the femoral head: a differential diagnosis in acute onset of coxarthrosis in the elderly. Arthritis Rheum. 1999;42(12):2719-23.

3. Yamamoto $T$, Schneider R, Bullough PG. Insufficiency subchondral fracture of the femoral head. Am J Surg Pathol. 2000;24(3):464-8.

4. Yamamoto T, Iwamoto Y, Schneider R, Bullough PG. Histopathological prevalence of subchondral insufficiency fracture of the femoral head. Ann Rheum Dis. 2008;67(2):150-3.

5. Rafii M, Mitnick H, Klug J, Firooznia H. Insufficiency fracture of the femoral head: MR imaging in three patients. AJR Am J Roentgenol. 1997;168(1):15963.

6. Hagino H, Okano T, Teshima R, Nishi T, Yamamoto K. Insufficiency fracture of the femoral head in patients with severe osteoporosis--report of 2 cases. Acta Orthop Scand. 1999;70(1):87-9.

7. Motomura G, Yamamoto T, Miyanishi K, Shirasawa K, Noguchi Y, Iwamoto Y. Subchondral insufficiency fracture of the femoral head and acetabulum : a case report. J Bone Joint Surg Am. 2002;84(7):1205-9.

8. Sonoda K, Motomura G, Ikemura S, Kubo Y, Yamamoto T, Nakashima Y. Favorable clinical and radiographic results of transtrochanteric anterior rotational osteotomy for collapsed subchondral insufficiency fracture of the femoral head in young adults. JB JS Open Access. 2017;2(1):e0013.

9. Suri S, Walsh DA. Osteochondral alterations in osteoarthritis. Bone. 2012 51(2):204-11.

10. Li G, Yin J, Gao J, Cheng TS, Pavlos NJ, Zhang C, Zheng MH. Subchondral bone in osteoarthritis: insight into risk factors and microstructural changes Arthritis Res Ther. 2013:15(6):223.

11. Yamamoto T, Nakashima Y, Shuto T, Jingushi S, Iwamoto Y. Subchondral insufficiency fracture of the femoral head in younger adults. Skelet Radiol. 2007;36(Suppl 1):S38-42

12. Iwasaki K, Yamamoto T, Motomura G, Mawatari T, Nakashima $Y$, Iwamoto $Y$. Subchondral insufficiency fracture of the femoral head in young adults. Clin Imaging. 2011;35(3):208-13.

13. Yoon PW, Kwak HS, Yoo JJ, Yoon KS, Kim HJ. Subchondral insufficiency fracture of the femoral head in elderly people. J Korean Med Sci. 2014;29(4): 593-8.

14. Yamamoto T, Karasuyama K, Iwasaki K, Doi T, Iwamoto Y. Subchondral insufficiency fracture of the femoral head in males. Arch Orthop Trauma Surg. 2014;134(9):1199-203.
15. Miyanishi K, Ishihara K, Jingushi S, Torisu T. Risk factors leading to total hip arthroplasty in patients with subchondral insufficiency fractures of the femoral head. J Orthop Surg (Hong Kong). 2010;18(3):271-5.

16. Iwasaki K, Yamamoto T, Motomura G, Ikemura S, Mawatari T, Nakashima Y, Iwamoto Y. Prognostic factors associated with a subchondral insufficiency fracture of the femoral head. Br J Radiol. 2012;85(1011):214-8.

17. Iwasaki K, Yamamoto T, Motomura G, Karasuyama K, Sonoda K, Kubo Y, Iwamoto $Y$. Common site of subchondral insufficiency fractures of the femoral head based on three-dimensional magnetic resonance imaging. Skelet Radiol. 2016;45(1):105-13.

18. Sonoda K, Yamamoto T, Motomura G, Karasuyama K, Kubo Y, Iwamoto Y Fat-suppressed T2-weighted MRI appearance of subchondral insufficiency fracture of the femoral head. Skelet Radiol. 2016:45(11):1515-21.

19. Yamamoto T. Subchondral insufficiency fractures of the femoral head. Clin Orthop Surg. 2012:4(3):173-80

20. Ishihara K, Miyanishi K, Ihara H, Jingushi S, Torisu T. Subchondral insufficiency fracture of the femoral head may be associated with hip dysplasia: a pilot study. Clin Orthop Relat Res. 2010;468(5):1331-5.

21. Ammann P, Rizzoli R. Bone strength and its determinants. Osteoporos Int. 2003;14(Suppl 3):S13-8.

22. Cox G, Einhorn TA, Tzioupis C, Giannoudis PV. Bone-turnover markers in fracture healing. J Bone Joint Surg Br. 2010:92(3):329-34.

23. Yamamoto $T$, Schneider R, Bullough PG. Subchondral insufficiency fracture of the femoral head: histopathologic correlation with MRI. Skelet Radiol. 2001;30(5):247-54

24. Yamamoto $T$, Takabatake $K$, Iwamoto $Y$. Subchondral insufficiency fracture of the femoral head resulting in rapid destruction of the hip joint: a sequential radiographic study. AJR Am J Roentgenol. 2002;178(2):435-7.

25. Ikemura S, Mawatari T, Matsui G, Iguchi T, Mitsuyasu H. Clinical outcomes in relation to locations of bone marrow edema lesions in patients with a subchondral insufficiency fracture of the hip: a review of fifteen cases. $\mathrm{Br}$ Radiol. 2016;89(1066):20150750

26. Matsuo K, Hirohata T, Sugioka Y, Ikeda M, Fukuda A. Influence of alcohol intake, cigarette smoking, and occupational status on idiopathic osteonecrosis of the femoral head. Clin Orthop Relat Res. 1988:234:115-23.

27. Vande Berg BC, Malghem J, Goffin EJ, Duprez TP, Maldague BE. Transient epiphyseal lesions in renal transplant recipients: presumed insufficiency stress fractures. Radiology. 1994;191(2):403-7.

28. Song WS, Yoo JJ, Koo KH, Yoon KS, Kim YM, Kim HJ. Subchondral fatique fracture of the femoral head in military recruits. J Bone Joint Surg Am. 2004; 86-A(9):1917-24.

29. Ishizu H, Shimizu T, Kaibara T, Asano T, Terkawi MA, Takahashi D, Iwasaki N. Bilateral subchondral insufficiency fractures of the femoral head in a normally active adolescent patient: a case report. J Orthop Sci. 2019:509492658(19)30148-4.

30. Uchida S, Noguchi M, Utsunomiya H, Kanezaki S, Mori T, Matsuda DK, Sakai A. Hip arthroscopy enables classification and treatment of precollapse subchondral insufficiency fracture of the femoral head associated intraarticular pathology. Knee Surg Sports Traumatol Arthrosc. 2018;26(8):2527-

\section{Publisher's Note}

Springer Nature remains neutral with regard to jurisdictional claims in published maps and institutional affiliations.

Ready to submit your research? Choose BMC and benefit from:

- fast, convenient online submission

- thorough peer review by experienced researchers in your field

- rapid publication on acceptance

- support for research data, including large and complex data types

- gold Open Access which fosters wider collaboration and increased citations

- maximum visibility for your research: over $100 \mathrm{M}$ website views per year

At BMC, research is always in progress.

Learn more biomedcentral.com/submission 